\title{
Automated Generation of Physical Surrogate Vehicle Models for Crash Optimization
}

\begin{abstract}
A challenge in the design and optimization of vehicle structures is the high computational costs required for crash analysis. In this paper an automated model generation for simplified vehicle crash models is presented. The considered crash load cases are the US NCAP $(100 \%, 56 \mathrm{~km} / \mathrm{h})$, the Euro NCAP $(40 \%, 64 \mathrm{~km} / \mathrm{h})$ and the IIHS Small Overlap $(25 \%, 64 \mathrm{~km} / \mathrm{h})$. The generation of the physical surrogate vehicle models is based on different sub-steps which were automated using a process chain. With this process chain it is possible to evaluate very efficiently the influence of structural modifications on the global crash behavior. During the model generation the crash behavior of the surrogate model is directly compared with the full vehicle model to enable a direct assessment of the model quality. Since the interface, where the model is cut, is an important factor for the obtained correlation, different interface positions were analysed. With obtained solutions it is possible to identify the interface position, which fulfils the required correlation by a given computational time. Additionally, the interface discretisation is analyzed to identify the model configuration with the highest correlation. This investigation was performed for three different vehicle models.
\end{abstract}

Keywords

Crashworthiness, Physical Surrogates, Simplified Models, Automated Model Generation, Optimization, Computational Time

\section{Introduction}

Vehicles body structures have to be lightweight with high crash safety to protect occupants and pedestrians. The development of a light and crash safe body structure is currently supported by simulations and structural optimizations. Due to the increased application of virtual methods the product development process for a new vehicle has been more than halved leading to shorter product life cycles [7], [15]. However, due to the high computational costs required for crash simulations, structural optimizations cannot be carried out with a full vehicle model. Especially since various crash load cases have to be considered for the development of a body structure. The influence of the crash load cases on the computational costs are described in [16]. In the case of a full vehicle optimization, the time required to obtain the optimization results can quickly increase to several weeks [7]. To counteract this problem, various 
countermeasures are described, such as increasing the number of CPUs or selecting a better optimization algorithm. Another option for reducing the computational time is through the use of surrogate models [29].

According to [6], physical surrogate models can be subdivided into eight modelling approaches. The simplification methods for vehicle crash models important for the developed modelling approach are the hybrid nonlinear FE-rigid approach, the sub-structure modelling approach and the multi-body system approach. In the hybrid nonlinear FE-rigid approach a part of the full model is set rigid. [27] Therefore, the elastic deformation and possible structural failure of the rigidified parts are neglected, which significantly reduces the computational costs. In the hybrid nonlinear FE-elastic approach the complex material behavior are replaced by a purely elastic material description. [6] In [1], [9] and [31] computational time reduction is obtained by applying special interface conditions in the sub-structure modelling approach e.g. applying guided deformation histories to the interface nodes of a sub-model or by removing structural parts of the vehicle model (e.g. in case of a front crash the whole rear structure of the vehicle). The responses of such simplified models are relatively correct as long as the deformation behavior of the sub-structure changes only slightly. [6]

The multi-body system approach represents a very simple but effective simplification technique; hereby, the structural behavior of all structural parts are represented by simplified mass-spring-damper systems, e.g. [12], [9], [13], [5], [17], [11], [22], [18] and [8]. This modelling method is particularly well suited for parametric studies during the conceptual design phase in the early product development process. The structural properties have to be determined experimentally or adjusted iteratively in order to reproduce the required structural behavior. However, the identification of structural parts with the required deformation characteristics can be a significant challenge. Still, the multi-body system approach can be used to estimate the loads on the vehicle passengers in the early development process [4], [21].

Another modeling approach is the FE beam model, as described in [24], [14] and [23]. Individual parts are represented as kinetic and kinematic descriptions. In this description the deformation characteristics of entire structures are replaced by macro elements. The required characteristics can be determined by means of 
experiments [2], detailed simulations [25] or analytical correlations [8]. The disadvantage of this approach is the fact that the identification of structural design concepts from obtained stiffness values is challenging.

By combining two or more simplification approaches from [6] a new approach is obtained. Hereby the combination of sub-structure modelling- and multi-body system approach is often used. This approach is characterized by cutting the vehicle structure and replacing the removed parts by lumped masses connected via rigid, elastic or nonlinear spring-damper elements or beam systems. In [10] the number of elements in a FE vehicle model is reduced by removing the rear structure of the vehicle behind the B-pillar and replacing the masses and inertial properties of the removed components with a rigid body. The rigid body is connected using the vehicle's load-bearing structures. In this way, the crash kinematics of the full vehicle are modelled; however, direct application of the masses and inertial properties of the crash model to the rigid body description is not possible, as a different deformation behavior of the body structure is obtained. Correct deformation behavior is only obtained after adapting the mass and the inertial properties of the rigid body [10]. For the structural optimization of a crash box, in [28] and [30] the full vehicle crash model is reduced by replacing all crash-irrelevant components (e.g. rear structure of body-in-white, doors and interior) with rigid bodies with corresponding mass and inertial properties. Subsequent optimization of the masses and inertial properties was also necessary to model the deformation behavior, as the initial deviation of the internal energy in the crash box was $\sim 25 \%$ between the full car and the surrogate model. After calibrating the rigid body properties in [28], a 90 \% computational time reduction could be achieved with a deviation of $5.4 \%$. The approach in [26] uses global deformation characteristics of structures which are obtained from the global crash model. For the simplification of the vehicle crash model, structural regions which sustain only elastic deformations during the frontal crash are replaced by kinematic numerical representations which describe both stiffness and load paths at the interface of the substituted structures. Within a four step approach, the characteristics to model the global deformation behavior are determined which are required to generate a physical surrogate description of the vehicle crash model. These characteristics include the mass, center of gravity of the structure to be replaced, the force pulse distribution in the interface and the 
stiffness of the replaced body structure in the longitudinal direction. One particular feature in positioning the spring-damper elements arises from the vectorial orientation of these elements in space. Due to that complex springdamper element positioning process it is challenging to automate.

In this paper a slight modified approach is presented, which allows in comparison to [26] an automated generation of physical surrogate models. Due to the high grade of automation, it is possible to investigate different interface positions and interface configurations, which is necessary to identify the interface definitions with the required correlation and calculation time.

\section{Automated generation of simplified vehicle models}

The physical background of the surrogate model generation is described in detail in [26]. Therefore, only a short overview is given in this paper. To generate a simplified description of the vehicle crash model the global deformation characteristics has to be determined. These characteristics include the mass, center of gravity of the structure to be replaced, the force pulse distribution in the interface and the stiffness of the replaced body structure in the longitudinal direction.

For the automation the model generation, as described in [26], had to be slightly adapted. The main difference is the orientation of the spring-damper-elements, representing the stiffness distribution of the replaced structure. In [26] those elements were oriented according to the orientation of the replaced body parts. The orientations of all elements were aligned according to the longitudinal direction of the vehicle. The main difference between these two models can be seen in the orientation of the spring/beam elements representing the stiffness of the A-pillar. In the left model in Fig. 1 the spring elements are oriented according to the orientation of the body parts in the cut section and the beam element in the right model in Fig. 1 are only oriented in x-direction. Hence, only the force in longitudinal direction is considered for the determination of the force pulse distribution in the interface. The effect of the applied simplification can be seen in the difference of the obtained force pulse distributions, as shown in Figure 1. The good correlation between these two models directly indicates that the obtained crash results will be very similar. This support the assumption, that this approach is acceptable for a simplified automation process. As in [26] the main 
advantage of this modelling approach is the reduction in computational time for performing a crash simulation due to the significantly reduced number of elements.

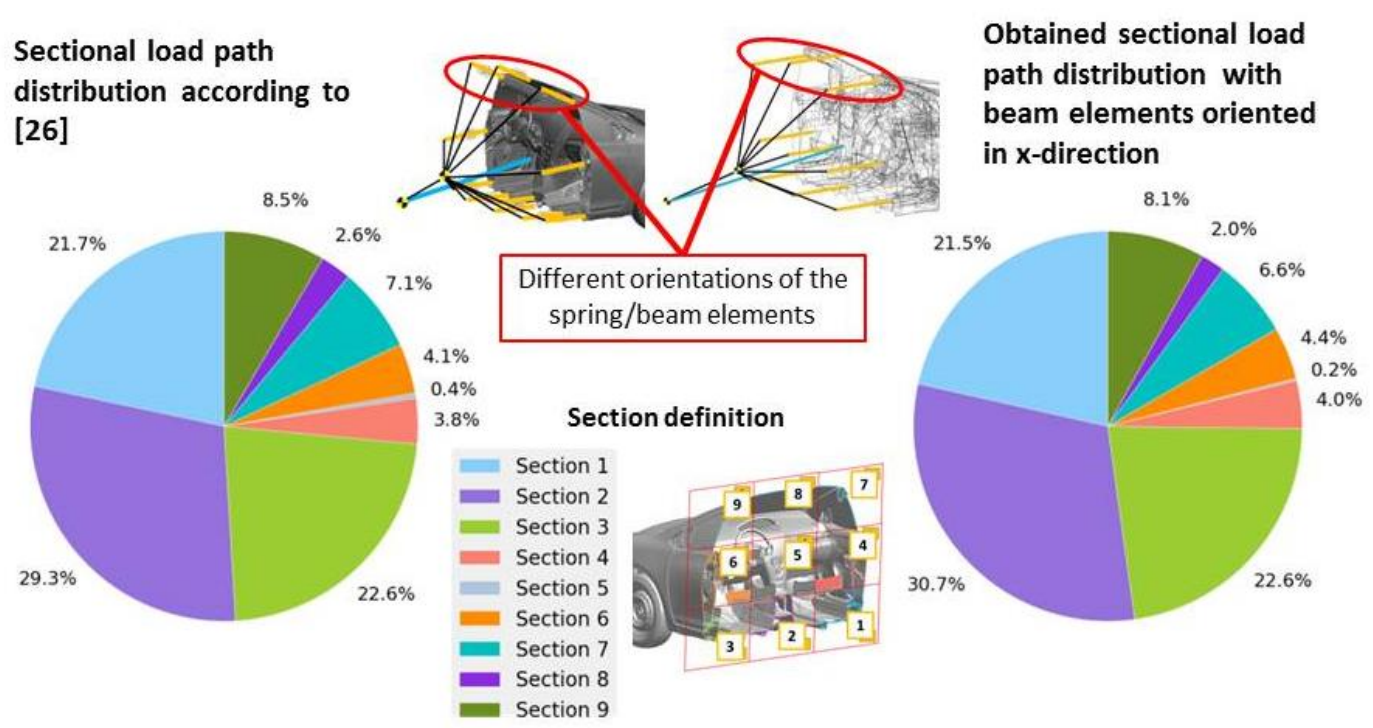

Fig. 1: Crash load distributions for resultant - and simplified longitudinal - element orientations [Yaris vehicle model - cross section position $\mathrm{x}=-1600 \mathrm{~mm}$ - US NCAP $(100 \%, 56 \mathrm{~km} / \mathrm{h})$ ]

For a further reduction of computational time, an automated four-step approach for the generation of a simplified vehicle model was developed. For the automation the generation of a simplified vehicle model is structured in four modules. The different modules and the used tools for the development are shown in Figure 2. Eventually the modules are linked to build up the final automation process.

The four modules are the analysis of the full vehicle model -, the analysis of the replaceable vehicle structure -, the modeling of the simplified vehicle structure and the post processing module. The process chain is realized using different tools. Python is used to operate the complete process chain and for some evaluation e.g. the extraction of cross section data or the calculation of the force distributions. The post processing module is entirely written in python to generate the comparative plots between the surrogate model and the complete crash model. With this plots the correlation quality of the simplified crash model is obtained directly. All simulations were performed using LS-DYNA version 7.1.2.

Before initiating the model generation, certain input is required as the position of the interface, the discretization of the interface (number of elements) and the part IDs for which the post processing should be applied on (correlation assessment). 
Before initiation also a node set has to be defined for the subsequent application of boundary condition in the analysis of the replaceable vehicle structure module. In the following the individual modules are described in more detail.

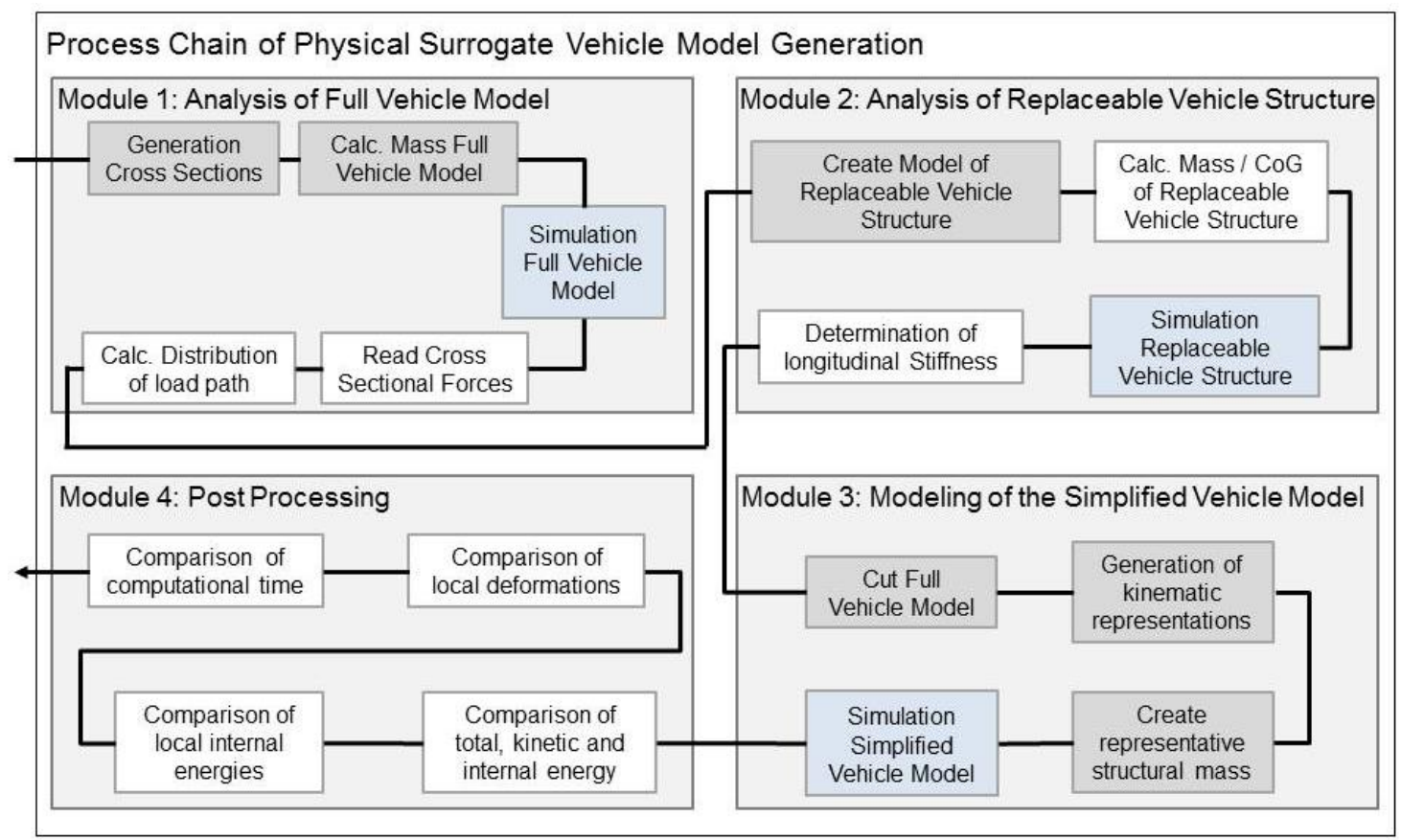

Fig. 2: Process chain of the surrogate model generation

\section{Module 1: Analysis Full Vehicle Model}

Module 1 is the analysis module of the full vehicle model. A full vehicle crash simulation is performed with a defined number of cross sections at the defined interface position. The number of cross section corresponds to the interface discretization configuration. In Figure 3 the generated sections for the interface are shown. In this model the interface was discretized with three elements in horizontal - and three elements in vertical direction [ $3 \times 3$ model]. For these sections the longitudinal-force-time characteristic curves are extracted as an output result from the global crash analysis. The impulse-time characteristic curves are obtained by integration of the $\mathrm{x}$-force-time characteristic curves (all coordinate systems are located at the middle of the front axle), which is used for the quantification of the load-path distribution [26]. Figure 4 shows the load-path distributions for the US NCAP $(100 \%, 56 \mathrm{~km} / \mathrm{h})$ crash load cases. The results show, that the most significant loads are obtained in section 1 to 3 which corresponds to the tunnel (30.7\%) and door sill structures $(21.5 \%$ and $22.6 \%)$. 


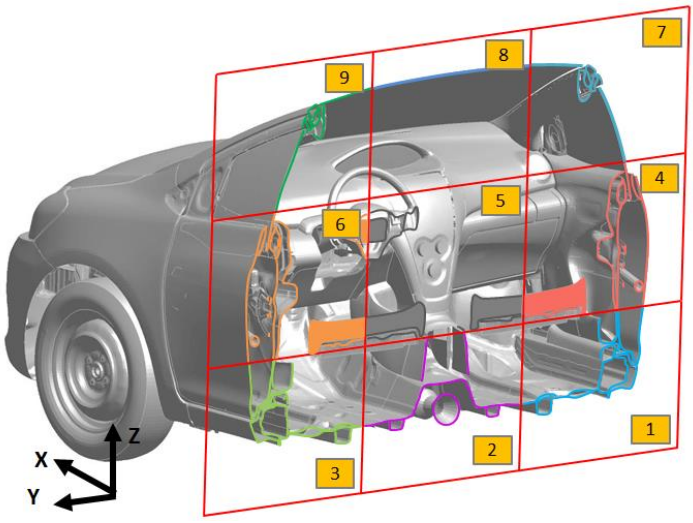

Fig. 3: Generated cross sections for an interface discretization with three elements in horizontal direction and three elements in vertical direction (interface at $\mathrm{x}=-1600 \mathrm{~mm}$ )

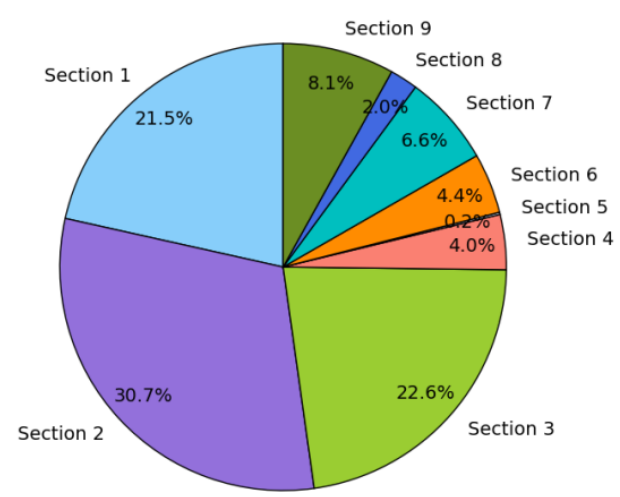

Fig. 4: Load distribution in the interface $(x=-$ $1600 \mathrm{~mm}$ ) at US NCAP crash load case (100\%, rigid wall, $56 \mathrm{~km} / \mathrm{h}$ impact speed)

The obtained load-path distribution is required in module 3 for the subsequent generation of the simplified vehicle model, since the global longitudinal stiffness of the vehicle, calculated in module 2 is split according to the obtained distribution. Additionally the total mass of the vehicle is calculated to obtain the identical model mass for the surrogate model in module 3.

\section{Module 2: Analysis of Replaceable Vehicle Structure}

In module 2 the cumulative center of gravity $(\mathrm{CoG})$ of the replaceable structure and the center of the connecting points (rear axle to car body) is determined. In the performed simulation the influence of joints, rubber bearings as well as interior parts, which also contribute slightly to the global stiffness, are taken into account. In Figure 5 the connecting points of the rear axle and the rigid body element at the interface, used for load introduction, are shown. At the interface a force of $1000 \mathrm{~N}$ in longitudinal direction (Fig. 5 red arrow) is applied with a rigid-body element and an explicit structural simulation is performed. The force was selected to have an elastic deformation of the car. On the other hand the force is large enough to reduce the influence of nonlinearities obtained by local joint deformations. Since the stiffness is obtained by a quasi-static explicit simulation, no additional implicit vehicle model is required for the automated model generation. 
The obtained displacement behavior of the car body is finally saved as output. For the investigated vehicle model and the defined interface position an elastic longitudinal stiffness of $24676.7 \mathrm{~N} / \mathrm{mm}$ is obtained.

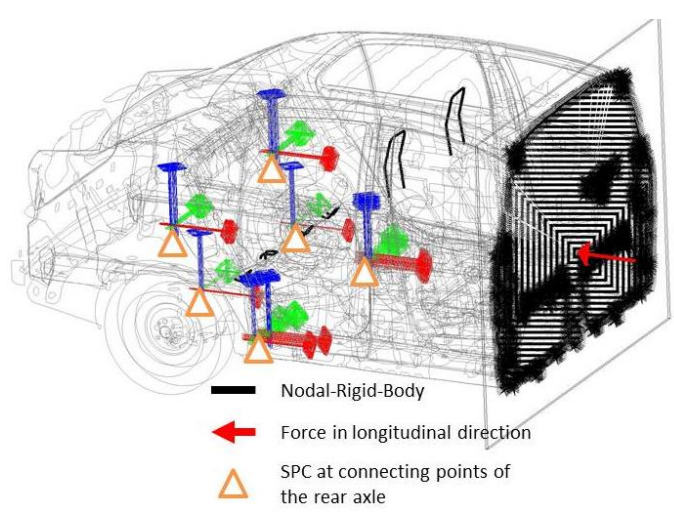

Fig. 5: FE-model to determine the longitudinal stiffness of the replaced vehicle structure

\section{Module 3: Modeling of the Surrogate Vehicle Model}

All the previous determined parameters (load-path distribution, cumulative CoG, longitudinal stiffness, the center of the rear axle and total mass of the vehicle) are finally used in module 3 to generate the surrogate vehicle model. First the vehicle is trimmed at the defined interface position and the elements for the kinematic description are applied as shown in Figure 6. Therefore the nodes of these beam elements are located in the center of gravity of each section, as shown in Figure 6. Additional one nodal rigid body is created at the interface position with its origin at the center of gravity of the corresponding interface. Eventually, this center of gravity is used for one element between the interface and the center of the rear axle. This additional element prevents rotational distortion of the vehicle structure during crash.

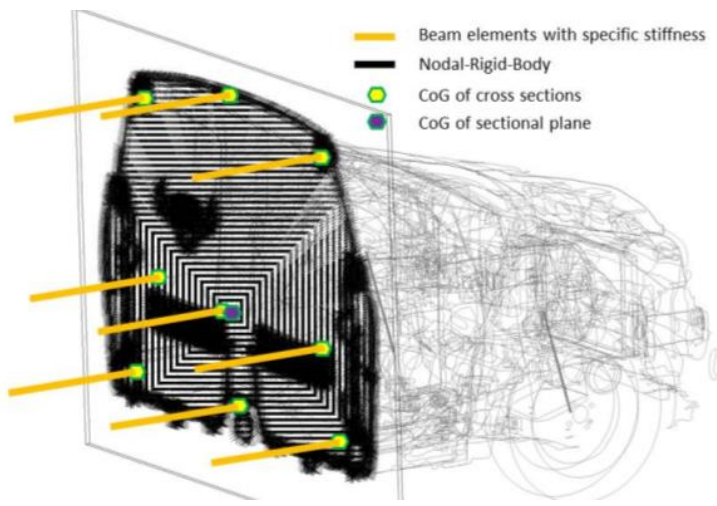

Fig. 6: FE-model of a surrogate vehicle crash model

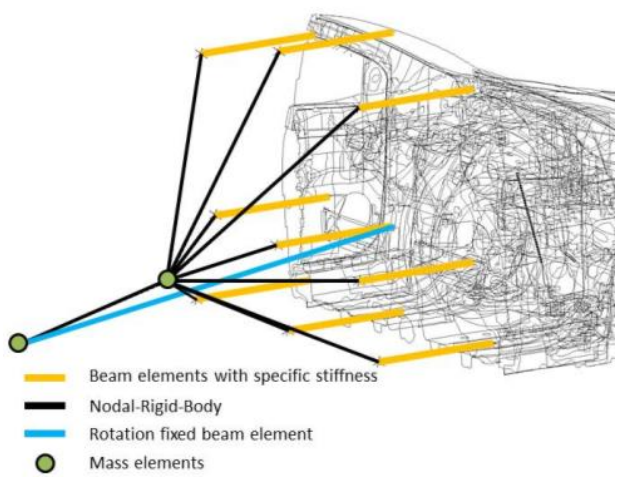

Fig. 7: Complete FE-model of a surrogate vehicle 
with the numeric representations for the trimmed

crash model

structures

The beam elements of the surrogate model (Fig. 6 yellow elements) are oriented along the longitudinal orientation of the vehicle. After the generation of the element, the substituted mass of the replaced structure is generated in the cumulative center of gravity. Finally, the elements for the distribution of the stiffness, the additional element to prevent rotational distortion and the substituted mass are connected together with a nodal rigid body, as shown in Figure 7.

\section{Module 4: Post Processing}

Module 4 is an optionally feature for the verification of the generated surrogate vehicle model. This module allows a direct comparison of energies and obtained deformation between the full vehicle model and the simplified crash model.

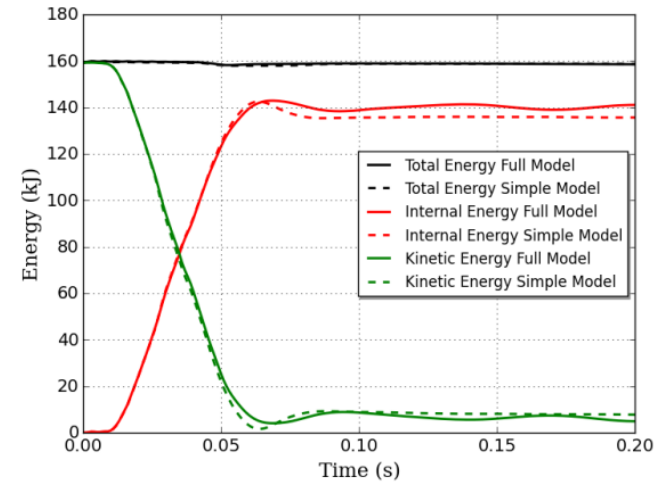

Fig. 8: Comparison of total, kinetic and internal energy for full and surrogate vehicle model at US NCAP crash load case (100\%, rigid wall, 56 km/h impact speed, vehicle mass $1263 \mathrm{~kg}, 5 \%$ mass scaling)

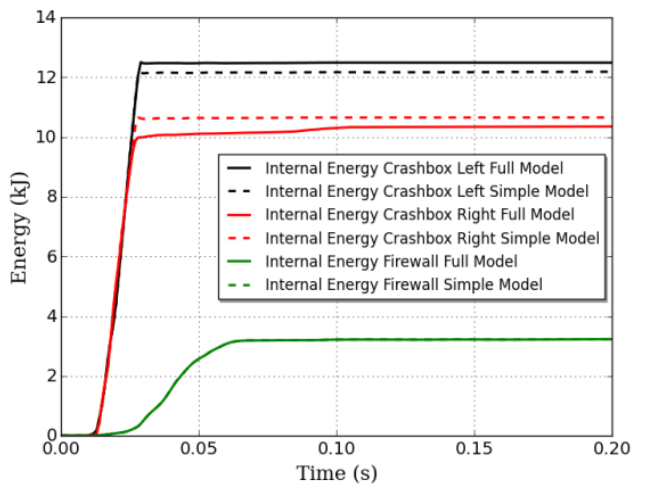

Fig. 9: Comparison of internal energy of crash box and firewall for full and surrogate vehicle models at US NCAP crash load case (100\%, rigid wall, $56 \mathrm{~km} / \mathrm{h}$ impact speed)

Thus the verification of the model is based on different levels; beside the comparison of the global energies (Figure 8), local deformation energies (Figure 9) and local deformations (Figure 10) are compared. In addition the computational time (Figure 11) is given as output to identify the model configuration with the required correlation by acceptable computational costs. As it can be seen in Figure 9, the deviation of the local energies between the models are low, which is sufficient to perform structural design studies using the surrogate vehicle model. 
In Figure 10 the deformed firewalls of both models are superimposed and the error in the firewall intrusion between these models is plotted for each node in the contour plot. Since the error between the intrusions is mainly below $4 \%$, the simplified vehicle model can model the intrusion behavior correctly.

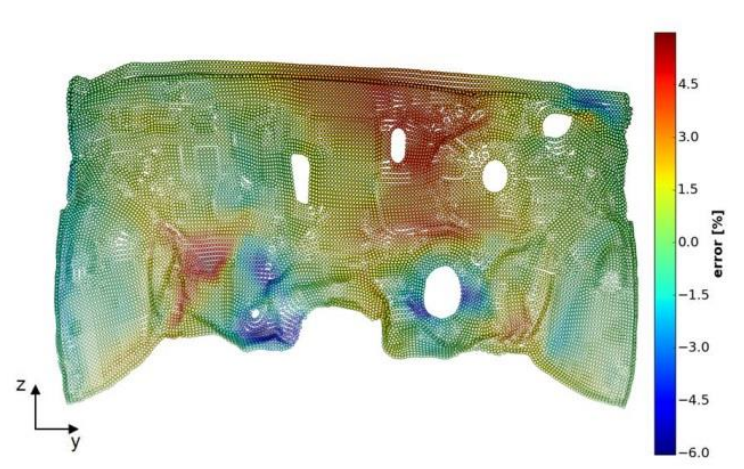

Fig. 10: Comparison of the local firewall deformation for full and surrogate vehicle model at US NCAP crash load case (100\%, rigid wall, $56 \mathrm{~km} / \mathrm{h}$ impact speed)

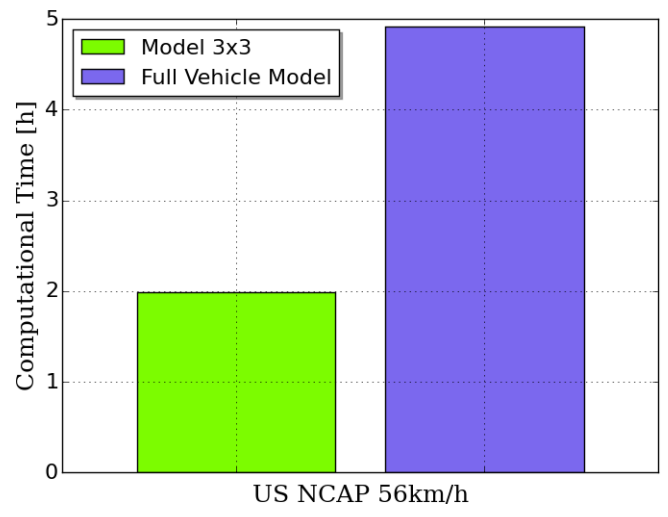

Fig. 11: Reduction of computational time through the use of the surrogate vehicle model $^{1}$

\section{Results}

With the described automated generation of the surrogate vehicle models it is possible to investigate different vehicle models, load cases, interface discretization configurations and positions of the interface. To investigate the influence of these parameters on the simulation results three vehicle FE crash models (Table 1) from the National Crash Analysis Center library and from the Centre for Collision Safety and Analysis were used. The results of this investigation are presented for the US NCAP $(100 \%, 56 \mathrm{~km} / \mathrm{h})$, Euro NCAP $(40 \%, 64 \mathrm{~km} / \mathrm{h})$ and the IIHS Small Overlap $(25 \%, 64 \mathrm{~km} / \mathrm{h})$ load cases.

The first model is the Toyota Yaris, which contains 1514068 elements [19]. This crash model was validated by comparing simulation results of acceleration pulses and structural deformations with crash tests. The second model is the Toyota Camry [3], which contains 2257280 elements. The FE model was validated by comparing several front and side crash tests with obtained simulation results. The third vehicle model is the Chevrolet Silverado. The model contains 963474 elements. This model was chosen due to its different global structural design [20].

${ }^{1}$ CASE2 Cluster - Intel "Ivy Bridge“" 12-core E5-2695v2 - 128 GB 1866MHZ DDR3 @ 2,4 GHz; 24 CPUs. 
The Silverado has a framework body-in-white design, which is different to the steel monocoque body-in-white design of Yaris or Camry.

With the Yaris model the influence of the interface position was investigated. The position of the interface correlates with the number of elements of the simplified vehicle model, which directly affects the quality of the model and the computational costs. Furthermore, the influence of the configuration of the interface was investigated by identifying the influence of the number of elements. Whilst the interface definition can have an influence on the model quality, it does not considerably affect the computational costs.

Tab. 1: Detailed list of the applied FE models

FE-Model
Toyota Yaris
(MY 2010)
[19]
[3]

\section{Influence of interface position on computational costs and model quality}

An important aspect when assessing the crash behavior of a structural concept is the local deformation of individual components or assemblies. A simplified vehicle model must represent these local deformation behaviors during crash with sufficient quality to avoid possible oversizing or even structural failure. If the local deformation of the firewall, for example, is not represented exactly in a surrogate crash model, an insufficient wall thickness can lead to structural failure 
when up-scaling the results into a full vehicle crash simulation. This should be avoided since the firewall secures the survival space of the passenger during the crash. Therefore, the influence of the interface position on the correlation of the firewall intrusion was investigated with a surrogate vehicle model of the Yaris with a configuration of three elements in horizontal - and three elements in vertical direction[ $3 \times 3$ model], as shown in Figure 7.

The identified dependency of the interface position on the computational costs is shown in Figure 12. By moving the interface position from $-2500 \mathrm{~mm}$ to $1200 \mathrm{~mm}$ in regard to the front of the vehicle, the computational time can be reduced by approximately $50 \%$. The obtained time reduction can play a significant role, if structural optimization has to be obtained.

For the assessment of the correlation quality, the averaged deviation in the obtained firewall intrusion into the passenger compartment was used. For each surrogate model the normalized correlation of the firewall is directly calculated using the following equation.

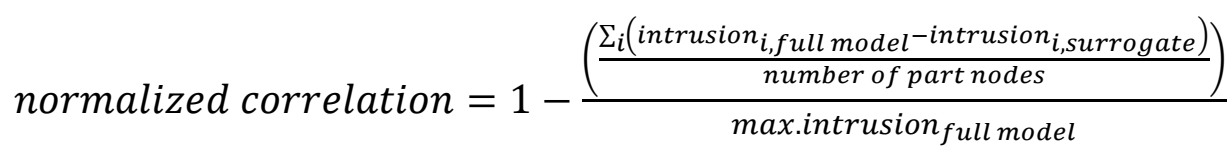

$$
\begin{aligned}
& (\mathrm{i}=1, \mathrm{n}(\mathrm{n}=\text { number of nodes })) \quad(1-1),
\end{aligned}
$$

In Figure 13 to 15 the obtained relation between the computational time and the correlation of the simplified model description is shown for the three investigated crash load cases - US NCAP (100\%, $56 \mathrm{~km} / \mathrm{h})$, Euro NCAP $(40 \%, 64 \mathrm{~km} / \mathrm{h})$ and IIHS Small Overlap $(25 \%, 64 \mathrm{~km} / \mathrm{h})$.

The results show a strong dependency of the model quality in regard to the interface position. As expected, the correlation gets better as further the interface is away from the deformed structures. On the other hand, an interface position too close to highly deformed structures leads to a decline in the correlation since the interface additional stiffens regions with high structural deformation. Whilst the quality of the model results decreases, the computational time per simulation goes down. Hence, based on the obtained solutions the surrogate model definitions can be adjusted to fulfill the specific requirements of a given optimization task in regard to computational costs and to the required correlation quality. 


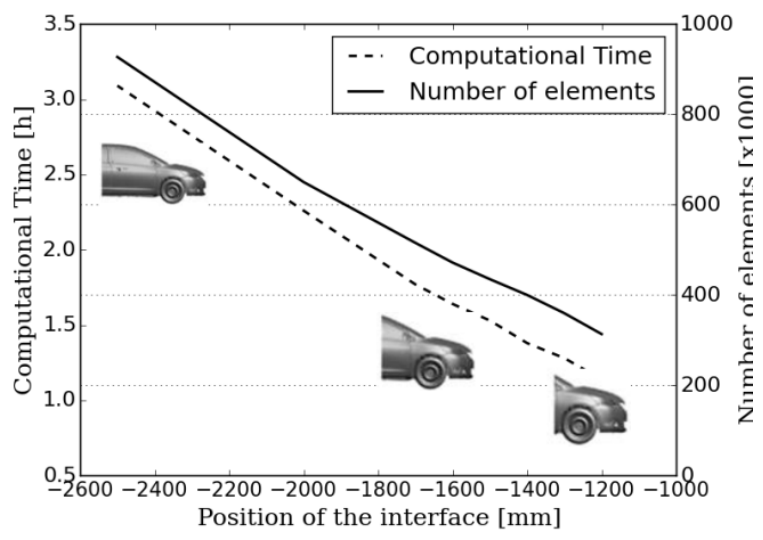

Fig. 12: Computational time reduction and number of elements in the dependence of the interface position [US NCAP crash load case ${ }^{1}$

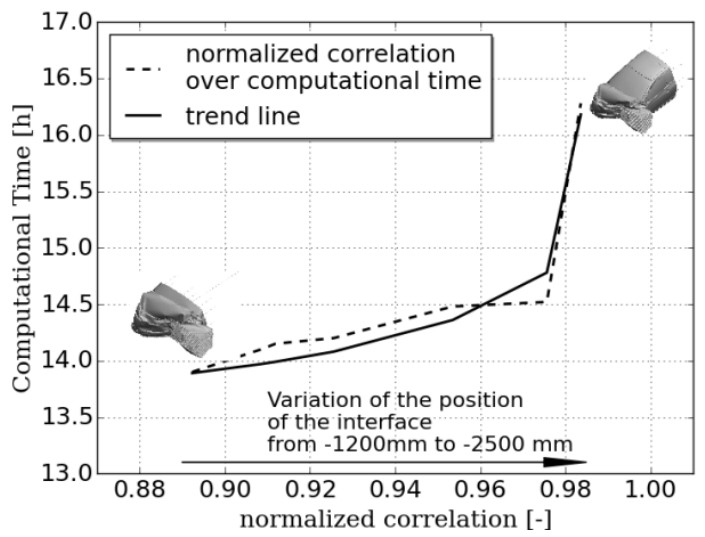

Fig. 14: Solutions for normalized correlation [firewall deformation] and computational time in the dependence of the interface position [EURO NCAP crash load case $^{1}$

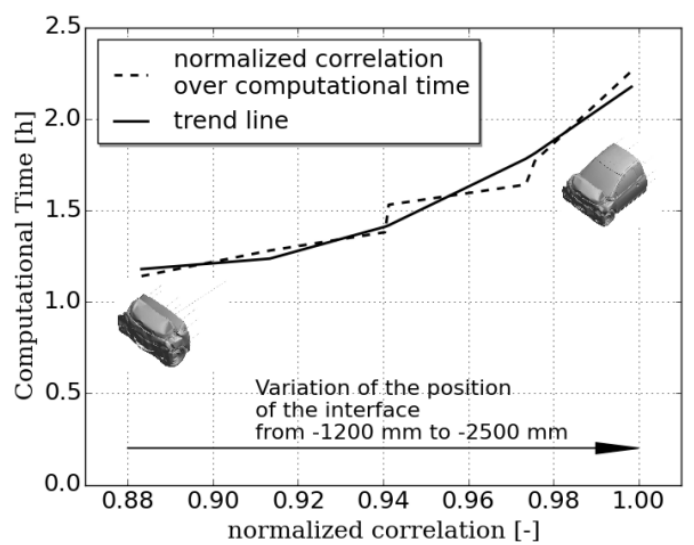

Fig. 13: Solutions for normalized correlation [firewall deformation] and computational time in the dependence of the interface position [US NCAP crash load case ${ }^{1}$

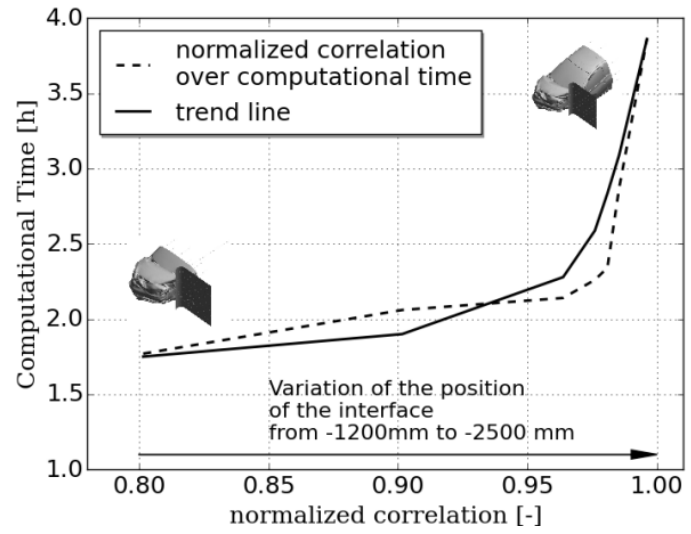

Fig. 15: Solutions for normalized correlation [firewall deformation] and computational time in the dependence of the interface position [IIHS Small Overlap crash load case ${ }^{1}$

\section{Investigation of the interface discretization}

Beside the interface position, the discretization of the interface, which corresponds to the number of element representations in the interface, is an important factor for the surrogate model. To identify the influence of different interface discretization sensitivity studies were performed at a constant interface position of $x=-1600 \mathrm{~mm}$. Four different interface configurations were investigated in this study. The first model (reference model) has an interface distribution in three elements in horizontal -and three elements in vertical direction. The other models correspond to an interface distribution of 1 - , $2 \times 2$ - and $4 \times 4$ - element representations. In figure 16 and 18 the different interface distribution of a model 
with a $2 \times 2$ element - and with the $4 \times 4$ element interface is shown. The different interface sections were numbered to show the relation between obtained loading and interface section of the vehicle. In Figure 17 and 19 the load-path distributions are shown for the $2 \times 2$ - and the $4 \times 4$ surrogate model. With a higher number of element representations a more detailed load flow through the car is obtained. However, similar loads are obtained, for a section of a model with coarse interface discretization and the sum of more sections representing the stiffness of the same region (for example section 2 [2x2 model] and the sum of section $3,4,7,8$ [4x4 model]).

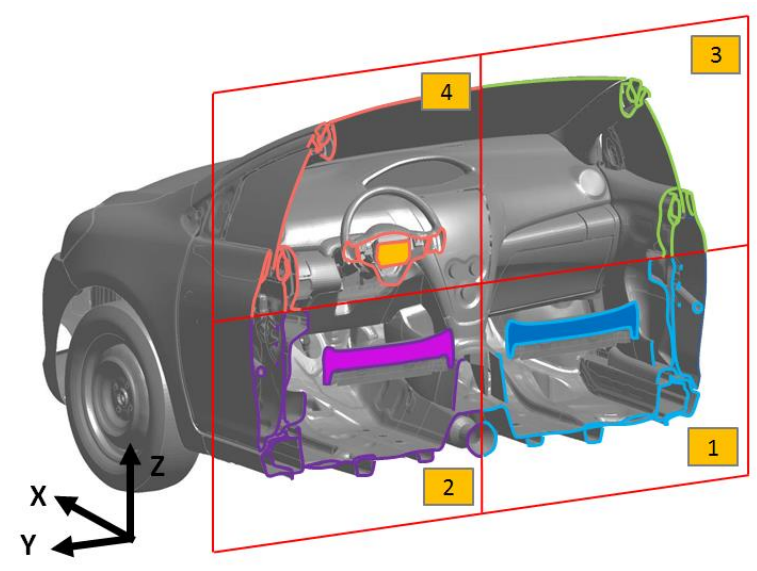

Fig. 16: Generated cross sections for an interface of two elements in horizontal direction and two elements in vertical direction $[2 \times 2$ model, $x=-$ $1600 \mathrm{~mm}]$

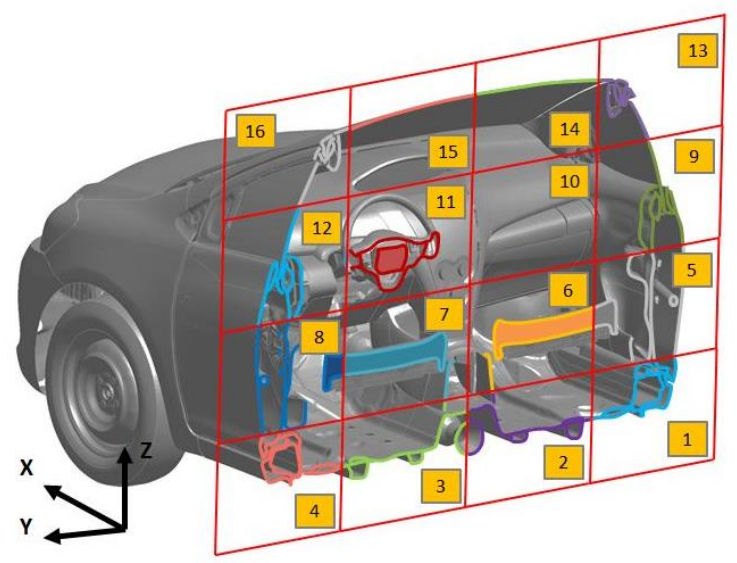

Fig. 18: Generated cross sections for an interface of four elements in horizontal direction and four elements in vertical direction [ $4 \times 4$ model, $x=-$ $1600 \mathrm{~mm}]$

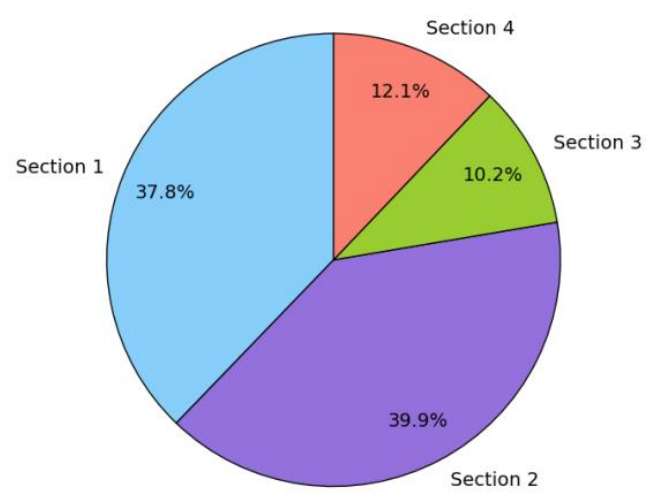

Fig. 17: Load path distribution for the $2 \times 2$ model at US NCAP crash load case [interface $x=-1600 \mathrm{~mm}$ ]

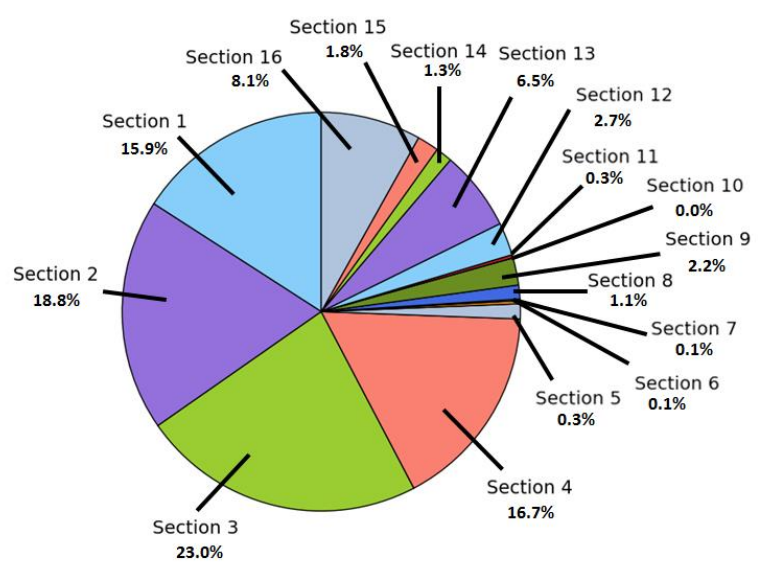

Fig. 19: Load path distribution for the $4 \times 4$ model at US NCAP crash load case [interface $x=-1600 \mathrm{~mm}$ ]

The number of elements included for surrogate model generation (like beam- and mass elements) has negligible influence on the computational costs since the time 
step of the elements is significantly higher than the time step of the elements of the vehicle. Figure 20 shows the computational time for the three investigated load cases for the full vehicle model and the different investigated surrogate models. As expected, the different interface discretization only has a negligible influence on the computational costs. On the other hand, the discretization of the interface affects the structural deformation during crash. The obtained correlations in the firewall deformation are shown for the different interface definitions in Figure 21. The results show, that the surrogate crash models can show a slightly different behavior for different interface definitions. In the study the best correlation between the firewall intrusions is obtained with the $3 \times 3$ model for the US NCAP, $4 \times 4$ for the Euro NCAP and $2 \times 2$ for the IHSS Small Overlap crash load case. In the investigation of the IHSS Small Overlap crash load case the Yaris vehicle rotated around the impactor during the crash. For the crash kinematics of a vehicle glancing off similar model accuracy can be assumed as long as the impactor is not too close to interface position with the rigidified areas. In this case the interface position has to be moved further to the rear of the vehicle to limit the influence of the rigidified areas. It is interesting that for each crash load case a different surrogate model configuration is required for an optimized surrogate model. This could be explained by the complexity of the deformation process during crash and the different grouping of structural parts into kinematic element representations.

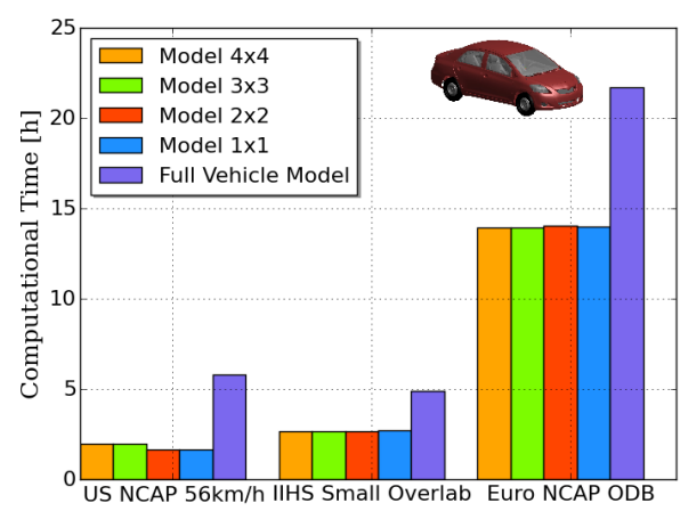

Fig. 20: Reduction of computational time for different Yaris surrogate models and different crash load cases 1

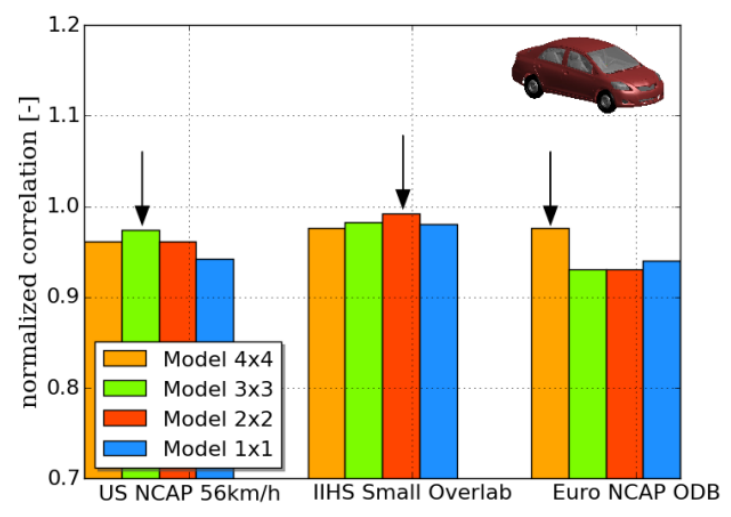

Fig. 21: Correlation of the firewall deformation for different Yaris surrogate models and different crash load cases 


\section{Verification of the surrogate model generation for different vehicle models}

Due to the complexity and the uniqueness of the crash behavior of a vehicle, the surrogate model generation has been investigated with other vehicle crash models. Surrogate models with different interface definitions were also generated from the crash models of the Toyota Camry and the Chevrolet Silverado. As for the Yaris model the discretization of the interface is negligible for the computational cost for the Camry and the Silverado. As shown in Figure 22 and Figure 24, the surrogate model is able to significantly reduce the computational time. However, as described before, the computational time reduction directly depends on the defined interface position.

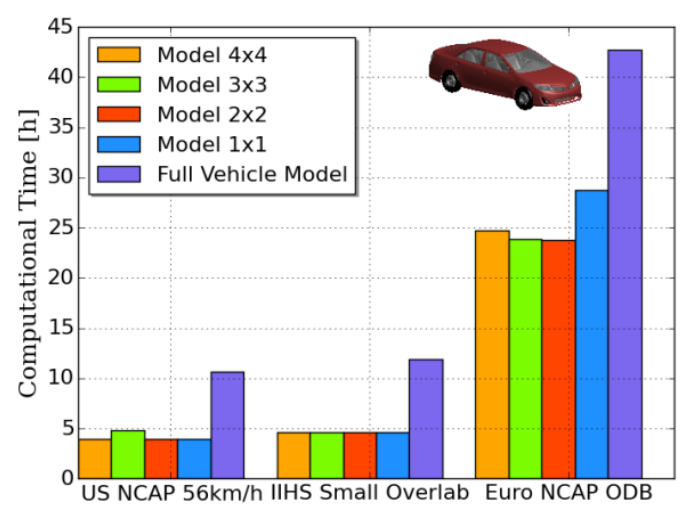

Fig. 22: Reduction of computational time for different Camry surrogate models and different crash load cases $^{1}$

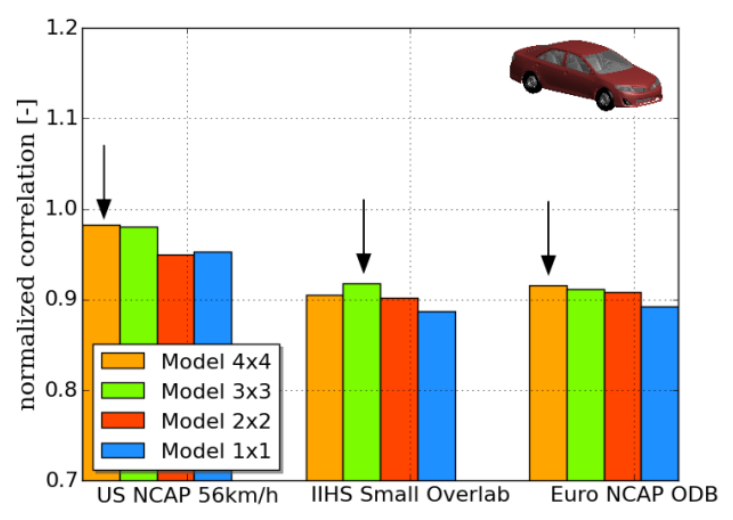

Fig. 23: Correlation of the firewall deformation for different Camry surrogate models and different crash load cases

The comparison of the firewall intrusions shows, that the best correlation of the firewall intrusion is also obtained for different interface definitions. The best correlation for the US NCAP crash load case is obtained for the Camry model as well as for the Silverado having a $4 \times 4$ element representation at the interface, which differs from to the result obtained for the Yaris [3x3]. In Figure 23 and Figure 25 the obtained correlations are shown for surrogate models of the Camry and the Silverado. The obtained results confirm that the optimum surrogate model configuration is strongly dependent on the deformation behavior of the vehicle and the investigated load case. Thus, a fixed recommendation for the interface definition cannot be given. Since the obtained correlation is always above $87 \%$, investigations can be performed with a predefined interface configuration. However, if the complete potential of this simplified vehicle description should be 
exploited, the optimum interface description has to be identified for each vehicle and each crash load case. The described automated model generation enables this identification process.

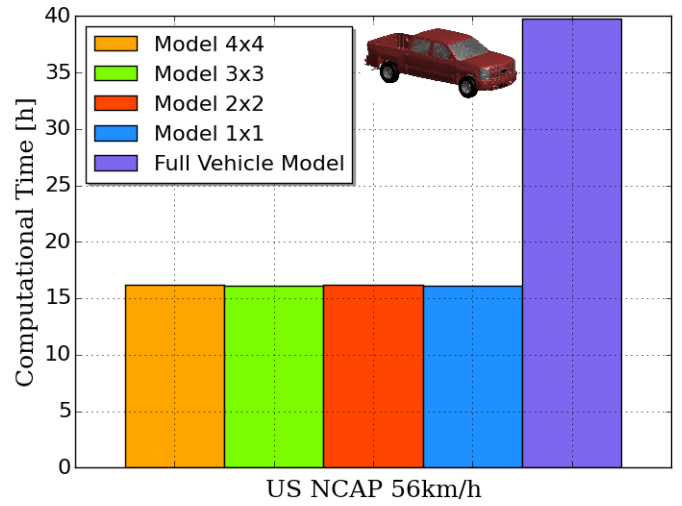

Fig. 24: Reduction of computational time deformation for different Silverado surrogate models for US NCAP crash load case $^{1}$

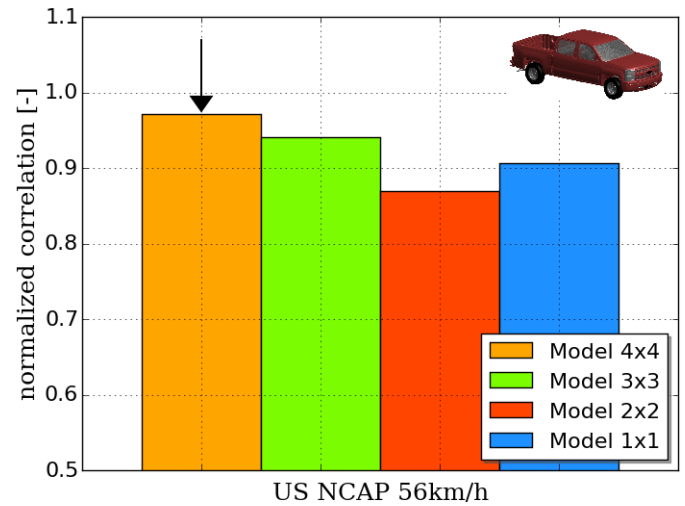

Fig. 25: Correlation of the firewall deformation for different Silverado surrogate models for the US NCAP crash load case

\section{Influence of the vehicle body-in-white design on the crash load distribution}

The load distribution during crash and the derived kinematic interface description are of significant importance for the quality of the surrogate crash model. If the rear of the vehicle is not available in the early conceptual phase, the load distribution can be derived from previous vehicle data if available. But if there are no previous data available, it can be difficult to set up a surrogate vehicle model. To be still able to generate a simplified crash model, the load distributions are summarized in Figure 26 for the US NCAP crash load case. The interface definition of Yaris [3x3], Camry [3x3] and Silverado [3x3] are identical as shown in Figure 3. The investigation shows that the load distribution strongly depends on the global body-in-white architecture of the vehicle.

Similar load distributions are obtained for the Yaris and Camry. This suggests that for a vehicle with conventional steel monocoque body-in-white design, in the first step a similar load distribution, as obtained for Yaris and Camry, can be assumed. However, this assumption cannot be applied on completely different body-inwhite architectures. Comparing the load distribution with the obtained results from the Silverado with its framework body-in-white architecture, the load distribution has completely changed and has to be adapted. Therefore, the 
presented surrogate model approach can be applied for the optimization of crash structures as soon as the load distribution of a vehicle with similar body-in-white design is known. Hence, the presented surrogate modelling approach is applicable in the early conceptual design phase as well as for serial development.

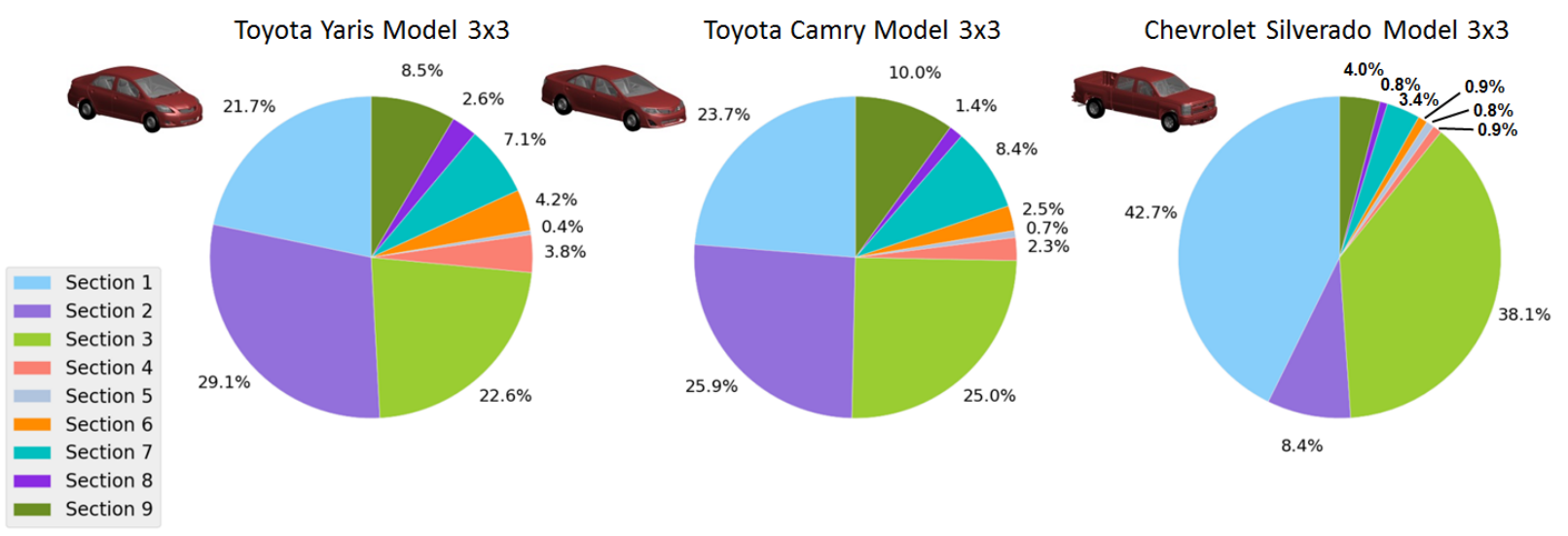

Fig. 26: Load path distribution for different vehicle crash models [US NCAP crash load case, $3 \times 3$ models, interface $\mathrm{x}=-1600 \mathrm{~mm}$, interface definitions as in figure 3 ]

\section{Conclusion}

The high computational costs for crash simulations are a significant challenge in the design process of vehicles, since the crash behavior has to be assessed for each derivative and equipment variant. Hence, optimization studies are currently difficult to perform due the high number of simulations required for the identification of the optimized structural design. In this paper, a novel automated surrogate modelling approach is presented for full vehicle models applicable for all frontal crash load cases.

With the developed process chain physical surrogate vehicle models can be generated to evaluate the crash behavior of different vehicle models and different load cases. Additionally, verification plots are generated to directly assess the correlation quality of the generated surrogate crash model. In this paper different interface positions were investigated to assess the influence of the position, where the vehicle is trimmed. With the obtained solutions it is possible to identify the surrogate model, which fulfils the given correlation requirements respectively to an optimization task. Furthermore, the interface discretization of the interface between detailed and simplified structural representation was analyzed to identify the interface configuration with the highest correlation in regard to the obtained firewall intrusion. This investigation was performed on three different vehicle 
models. In Table 2 the models with the best correlations are summarized results for the Toyota Yaris, the Toyota Camry and the Chevrolet Silverado. The results show that the interface definition with the highest correlation can differ between vehicles and crash load cases.

Tab. 2: Detailed list of the surrogate models for the three vehicle models and the load cases

\begin{tabular}{llll}
\hline FE Vehicle Model & US NCAP crash & Euro NCAP crash & IIHS Small Overlap \\
Toyota Yaris & $3 \times 3$ surrogate & $4 \times 4$ surrogate & 2x2 surrogate \\
& model & model & model \\
Toyota Camry & $4 \times 4$ surrogate & $4 \times 4$ surrogate & $3 \times 3$ surrogate \\
Chevrolet & model & model & model \\
Silverado & $4 \times 4$ surrogate & not examined & not examined \\
\hline
\end{tabular}

Using the presented simplified modelling approach, it is possible to significantly reduce the required computational costs for crash simulations. Due to the obtained time reduction it is possible to efficiently assess virtually the complex structural deformation during a vehicle crash. By applying the process chain for automated model generation, the position and the configuration of the interface between detailed and simplified modelling can be identified, which fulfils the required correlation by a given timeframe per simulation run. This assessment is feasible since correlation plots are directly created for each surrogate model. Therefore, the achievable computational time with the presented surrogate vehicle modelling approach could enables to integrate structural optimization in the design process of vehicle crash structures.

\section{Disclosure statement}

The research leading to these results received funding from the Helmholtz Association of German Research Centres within the research topic Next Generation Car. 


\section{References}

[1] Altair, RADIOSS: Sub-modeling (cut approach), http://www.altairuniversity.com/wpcontent/uploads/2012/08/RADIOSS_CRASH_CUT_V05.pdf, 2012.

[2] Beeh, E., Development of a novel crash-adaptive front-end structure for alternative driven vehicles, PhD thesis, German Aerospace Center, ISSN 1434-8454, 2015.

[3] CCSA: Development \& Validation of a Finite Element Model for the 2012 Toyota Camry Passenger Sedan, Georg Mason University, Faifax, 2016. DOI: $10.13021 / G 8 N 88$.

[4] Deb, A. and Srinivas, K. C., Development of a new lumped-parameter model for vehicle side-impact safety simulation, J. Automobile Engineering, Proc. IMechE, Vol. 222, Part D, 2008, DOI: 10.1243/09544070JAUTO801.

[5] Du Bois, P., Chou, C. C., Fileta, B. B., Khalil, T. B., King, A. I., Mahmood, H. F., Mertz, H. J. and Wismans, J., Vehicle Crashworthiness and occupant protection, American Iron and Steel Institue, Michigan, 2004.

[6] Duddeck, F. and Wehrle, E., Recent Advances on Surrogate Modelling for Robustness Assessment of Structures with respect to Crashworthiness Requirements, 10th European LS-DYNA Conference 2015, Würzburg, Germany, 2015.

[7] Duddeck, F., Multidisciplinary optimization of car bodies, Struct. Multidisc. Optim. 35 (2008), pp. 375-389, DOI: 10.1007/s00158-0070130-6.

[8] Fender, J., Solution Spaces for Vehicle Crash Design, PhD thesis, Technical University Munich, Chair of Computational Mechanics, Munich, Germany, 2013.

[9] Gandhi, U.N. and Hu, S.J., Data-based approach in modeling automobile crash, Int. J. Impact Engrg. 16(1) (1995), pp. 95-118, DOI: 10.1016/0734743X(94)E0029-U.

[10] Hilmann, J., On the Development of a Process Chain for Structural 
Optimization in Vehicle Passive Safety, PhD Thesis, Technical University Berlin, 2009, URN: urn:nbn:de:kobv:83-opus-22605.

[11] Jonsén, P., Isaksson, E., Sundin, K. G. and Oldenburg, M., Identification of lumped paramter automotive crash models for bumper system development, Int. J. Crashworth. 14(6) (2009), pp. 533-541, DOI: $10.1080 / 13588260902837262$.

[12] Kamal, M. M., Analysis and Simulation of Vehicle to Barrier Impact, SAE Paper No. 700414, 1970.

[13] Kim, C.H., Mijar, A.R. and Arora, J.S., Development of simplified models for design and optimization of automotive structures for crashworthiness, Struct. Multidisc. Optim. 22 (2001), pp. 307-321, DOI: 10.1007/PL00013285.

[14] Kim, H.-S., Kang, S.-Y., Lee, I.-H., Park, S.-H. and Han, D.-C., Vehicle Frontal Crashworthiness Analysis by Simplified Structure Modeling using Nonlinear Spring and Beam Elements, International Journal of Crashworthiness, 2:1, pp. 107-118, DOI: 10.1533/cras.1997.0038.

[15] Klaiber, M.: Use of innovative 3D printing technologies for flexible process chaining, 2. Technologietag Hybrider Leichtbau, Stuttgart, Germany, 2015.

[16] Kodiyalam, S., Yang, R.J., Gu, L. and Tho, C.-H., Multidisciplinary design optimization of a vehicle system in a scalable, high performance computing environment, Struct. Multidisc. Optim. 26 (2004), pp. 256-263, DOI: 10.1007/s00158-003-0343-2.

[17] Marler, R.T., Kim, C.-H. and Arora, J.S., System identification of simplified crash models using multi-objective optimization, Comput. Methods Appl. Mech. Engrg. 195 (2006), pp. 4383-4395, DOI:

10.1016/j.cma.2005.09.002.

[18] Mooi, H. G. and Huibers, J.H.A.M., Simple and effective lumped mass models for determining kinetics and dynamics of car-to-car crashes, Int. J. Crashworth. 5(1) (2000), pp.7-23, DOI: 10.1533/cras.2000.0120.

[19] NCAC, Extended Validation of the Finite Element Model for the 2010 Toyota Yaris Passenger Sedan, The George Washington University, 2012. 
[20] NCAC; Extended Validation of the Finite Element Model for the 2007 Chevrolet Silverado Pick-Up Truck. The George Washington University, 2012.

[21] Pahlavani, M. and Marzbanrad, J., Crashworthiness study of a full vehiclelumped model using parameters optimisation, Int. J. Crashworth. 20:6, 2015, pp. 573-591, DOI: 10.1080/13588265.2015.1068910.

[22] Pawlus, W., Nielsen, J.E., Karimi, H.R. and Robbersmyr, K.G., Mathematical Modeling and Analysis of a Vehicle Crash, 4th European Computing Conference, Bucharest, 2010, pp. 194-199, ISBN: 978-960474-178-6.

[23] Qin, Y., A concept to design fleet compatible vehicles for real accident environments, Fortschritt-Bereichte VDI. Reihe 12, Verkehrstechnik/Fahrzeugtechnik, VDI Verlag, Düsseldorf, 2011, ISBN: 3183737124.

[24] Relou, J., Methods for the development of crash-compatible vehicles, Berichte aus der Fahrzeugtechnik, PhD Thesis, Shaker, Aachen, 2000, ISBN: 382657804X.

[25] Schäffer, M., Münster, M., Sturm, R. and Friedrich, H.E.: Development of an optimised side crash concept for the battery-electric vehicle concept Urban Modular Vehicle, 14. LS-DYNA Forum, Bamberg, 2016.

[26] Schäffer, M.; Sturm, R.; Friedrich, H.E.: Methodological approach for reducing computational costs of vehicle frontal crashworthiness analysis by using simplified structural modelling. International Journal of Crashworthiness, 2017, ?https://doi.org/10.1080/13588265.2017.1389631

[27] Schmidt, F. and Pitzer, M.: Component models of vehicle structures for side crash load case calculation, Karosseriebautage Hamburg, Germany, 2012.

[28] Schwanitz, P., Sankarasubramanian, H., Werner, S.W., Göhlich, D., Chawla, A. and Mukherjee, S., Methodology for Multiparamter Optimization During the Concept Phase for Crash Relevant Vehicle Structures, 9. Weimar Optimierungs- und Stochastiktage, Weimar, 
Germany, 2012.

[29] Stein, M., Development of a methodology to improve the structural interaction in a car-to-car-frontal crash, PhD Thesis, Berlin, Germany, 2015.

[30] Sturm, R.; Schäffer, M. und Münster, M.: Development of a safe modular body structure for a battery electric driven urban vehicle. In: Progress in Mechanics and Materials in Design 2017. Lusolmpress S.A. Progress in Mechanics and Materials in Design, 2017, Albufeira, Portugal. ISBN 978989-98832-6-0.

[31] Weigert, D., Duddeck, F. and Schluder, H., Automatic Model Reduction by Exploitation of Knowledge from Pre-existing Simulations, 13. LS-DYNA Forum, Bamberg, 2014. 